\title{
Leaders paysans et autorités religieuses comme courtiers du développement en milieu rural sénégalais
}

Monika Salzbrunn

\section{(Q) OpenEdition \\ Journals}

Édition électronique

URL : http://journals.openedition.org/apad/801

DOI : 10.4000/apad.801

ISSN : 1950-6929

Éditeur

LIT Verlag

Édition imprimée

Date de publication : 1 mai 1996

Référence électronique

Monika Salzbrunn, «Leaders paysans et autorités religieuses comme courtiers du développement en milieu rural sénégalais », Bulletin de l'APAD [En ligne], 11 | 1996, mis en ligne le 03 juillet 2007, consulté le 08 septembre 2020. URL : http://journals.openedition.org/apad/801 ; DOI : https://doi.org/10.4000/ apad.801

Ce document a été généré automatiquement le 8 septembre 2020

Bulletin de l'APAD 


\title{
Leaders paysans et autorités religieuses comme courtiers du développement en milieu rural sénégalais
}

\author{
Monika Salzbrunn
}

1 Basée sur une étude empirique effectuée dans deux villages du bassin arachidier sénégalais en $1994^{1}$, la contribution met en relief les interactions entre chefs traditionnels, leaders paysans et autorités religieuses dans le contexte de projets de développement Non seulement au niveau régional (relations de l'ADAK, Association d'agriculteurs de la région de Kaolack, et de l'Union de Malem Hodar avec des autorités religieuses comme l'imam et le marabout), mais aussi au niveau du village, de nombreuses interpénétrations se manifestant p.ex. à la position d'une personne dans un réseau pouvaient être observées.

2 Des personnes qui étaient à la fois impliquées dans une organisation paysanne, dans une confrérie islamique et qui disposaient de contacts avec des bailleurs de fonds jouaient des rôles clefs dans la "distribution" de projets de développement. Etaient également remarquées des relations de parenté, dans un cas exemplaire entre le trésorier de l'association paysanne régionale et l'Imam du village, en plus de contacts fréquents avec le président du groupement paysan local qui, quant à lui, était le descendant du fondateur du village. Une coordinatrice régionale de la FONGS, qui était en contact avec plusieurs bailleurs de fonds et qui avait des relations avec des membres de confréries sert de deuxième exemple.

3 La stratégie de ces nouveaux courtiers du développement qui cherchent désormais un contact direct avec les bailleurs de fonds s'inscrit dans la logique des relations entre les intermédiaires traditionnels tels que les membres importants des confréries (surtout la Muridiya et la Tidjaniya) et les représentants de l'Etat, s'adaptant de manière souple à des changements politiques (conséquences des programmes d'ajustement structurel; démocratisation), économiques (déclin de l'économie de l'arachide) et sociaux. Dans ce 
contexte, on peut se demander dans quelle mesure ces nouveaux courtiers sont partie intégrante des changements dans la société civile, en particulier en ce qui concerne le rapport entre les confréries et les organisations paysannes.

4 A partir d'un entretien avec N.A., Coordinatrice de la FONGS pour la promotion féminine à Kaffrine, centre urbain dans la région de Kaolack, on peut démontrer l'apprentissage du "discours ONG" et l'établissement d'une position stratégique dans l'enjeu de la politique de développement Après avoir travaillé dans l'administration comme animatrice, N.A. a accepté un poste à la FONGS (Fédération des Organisations Non-Gouvernementales du Sénégal), une organisation paysanne qui regroupe surtout les nombreux groupements villageois qui se sont crées les dix dernières années. En tant que Coordinatrice régionale pour la Promotion féminine, poste récemment crée, N.A. s'occupe davantage des groupes de femmes. Au moment où je fis sa connaissance, N.A. était en train d'évaluer un projet d'alphabétisation qui fait parti d'un projet soutenu par la Banque Mondiale et exécuté en collaboration avec la FONGS. Pour la première fois, je rencontrai N.A. lors d'un séminaire annuel de l'Union (niveau régional) des GIE (Groupement à Intérêt économique). Comme j'étais à la recherche d'un village dans lequel je pouvais effectuer une étude de terrain, N.A. se proposa comme intermédiaire. Mes contacts avec un projet de développement de la coopération bilatérale allemande, (P AGERNA, Projet d'Autopromotion et de Gestion de Ressources Naturelles) peuvent être une des raisons pour lesquelles N.A utilisait un vocabulaire faisant parti du "discours ONG" :

5 "C'est à dire les produits de ce champ collectif peuvent financer le moulin à mil ou équiper par exemple le puits le nouveau puits qu'on vient de creuser. Et il paraît que quand les gens ne pourront pas passer tout le temps à tirer la corde pour avoir de l'eau, comment trouver une pompe ou bien des méthodes beaucoup plus modernes pour afin d'alléger le poids à la portée des femmes."

6 Sachant qu'un puits et un moulin à mil sont des projets typiques soutenus en ce moment par des bailleurs de fonds, N.A. les utilise souvent comme exemple. En tant que Coordinatrice régionale pour la promotion féminine, elle s'intéresse évidemment à la situation des femmes. Mais c'est aussi parce que dans la politique actuelle de développement, ce sujet est très à la mode, qu'elle y revient sans arrêt Au début de l'interview, elle souligne la difficulté de discuter seulement avec les femmes d'un village. D'après elle, il faut toujours passer par les maris et par les marabouts. A l'aide de cette constatation, elle se montre indispensable en tant qu'agent qui introduit de nouvelles idées liées à de nouveaux projets :

$7 \quad \mathrm{~N}$ : Quand j'ai été appelée à la FONGS pour demander... pour être déléguée au niveau de cette chose là afin de mener une enquête, je suis passée d'abord par les marabouts. Je leur ai expliqué la situation. Je leur ai fait comprendre que comme tout le monde le dit, il n'y a jamais eu de développement sans formation. Et l'alphabétisation rentre dans le cadre de la formation quotidienne de la population. J'étais donc en contact avec eux, les marabouts, le chef de village, les différents notables et ils m'ont donné leur accord. Et vraiment, ils n'ont pas crée de difficultés à ce problème là. D'ailleurs ils l'ont accueilli à bras ouvert, ils ont beaucoup facilité la tâche ; d'ailleurs les différents auditeurs ont été (...) sur le même lieu quand je les ai appelé pour faire l'enquête. Entretemps ils ont préféré me donner le nom des auditeurs qui devaient suivre la classe d'alphabétisation - ce qui veut dire que les gens étaient vraiment motivés. 
M : Oui puis c'est comme ça.

M : Oui, j'ai vu.
M : Les noms des gens qui suivaient la classe? Ah oui. Mais j'ai discuté avec un autre marabout qui n'était vraiment pas d'accord avec ces idées-là

$\mathrm{N}$ : ça dépend des hommes (...) il y a des gens qui sont toujours résistants ; il faut faire rentrer ça dans leurs esprits.

$\mathrm{M}:$ il y a des changements des fois.

$\mathrm{N}$ : il y a des gens qui ne veulent pas le développement, ils refusent toute activité, toute sorte d'action donc... eux aussi ils sont mis de côté, hein?

$\mathrm{N}$ : Parce qu'on ne peut pas perdre de temps avec quelqu'un qui ne veut rien savoir qui ne veut rien comprendre, c'est pas possible ça.

M : Oui, justement, justement, mais...c'est vrai aussi qu'il y a des grandes différences entre les opinions des différents marabouts, mais...enfin, comme les gens ont l'habitude de leur demander toujours leur avis, c'est quand même important ce qu'ils disent (...)

$\mathrm{N}$ : Bien sûr, bien sûr. Parce que je ne veux pas qu'on m'interroge, dire 'je ne veux pas que tu viennes' avec notre matériel ou pour dire ce que nous allons planter, implanter une classe d'alphabétisation et qu'on n'a pas d'auditeurs ou bien si par exemple une mission de la FONGS manque, on ne peut plus la revoir quand les personnes ne sont plus en compte (...) Donc, avant toute activité, il faut d'abord prendre contact avec les gens, sensibiliser le terrain, et voir ce qui peut empêcher la réussite même de l'action et essayer de trouver des solutions appropriées à ce problème...C'est pour ça qu'on ne peut pas toujours entrer d'abord. A chaque fois, avant de mener quoi que ce soit, il faut d'abord prendre son temps avec les différents (types) de l'action et quand ils donnent leur avis et que cet avis n'est pas favorable attendre à nouveau à une (...) de l'action et

N.A. se montre alors comme une personne en laquelle les représentants différents du pouvoir (traditionnel, religieux etc.) ont confiance. En tant que membre de la confrérie des Mourides et descendante d'une famille de "rois locaux", elle peut compter sur l'écoute des marabouts. Chaque fois que je l'accompagnais dans un village, comme chez elle aussi, elle montrait l'importance de la pratique religieuse. Contrairement à la majorité des femmes, elle priait en public, en présence d'autres personnes. Souvent, elle déclarait à haute voix qu'elle avait encore des prières à "rattraper", se montrant soucieuse d'accomplir ses devoirs de musulmane. Dans son quartier, elle cherchait à acheter "le parfum du prophète", une sorte de gris-gris qui devait la protéger. Avant que je la quitte, elle m'en offrit aussi quelques pièces.

Parlant de ses expériences dans l'administration, elle affirme sa capacité de "travailler avec la population rurale", de connaître ses besoins et de défendre ses intérêts.

M : Mais est-ce que ce n'est pas difficile, en tant que femme, pour toi, de discuter avec eux ? Parce que, comme tu disais, c'est toujours la femme du mari...

9 A : Pas du tout, pas du tout, parce que la FONGS sait que j'ai travaillé même avant d'être au niveau de la FONGS. Je travaillais avec les populations, quand j'étais dans l'administration et je m'occupais des problèmes du monde rural. Donc j'étais habituée à rencontrer ces mêmes personnes (...) 
$21 \quad \mathrm{~A}$ :(...) C'est que je disais : avec le temps, ils arrivent à voir des résultats, et ainsi de suite, la FONGS (...) ils sont content. C'est pour ça que moi, je n'ai pas eu de problèmes au niveau de mon groupe. Sénégal, ces acteurs locaux occupent désormais une position clé en ce qui concerne les luttes politiques, le contrôle des ressources locales et le contrôle des financements extérieurs: "Lassés d'un Etat et d'une administration locale qui n'arrivent plus à entretenir convenablement leur clientèle rurale, les paysans se tournent de plus en plus vers d'autres sources de financement, notamment vers les institutions de développement nationales ou internationales (organisations non gouvernementales, agences de coopération bilatérale ou multilatérale, ambassades, organisations caritatives ou religieuses). C'est ainsi que s'affirme le rôle important et spécifique des nouveaux courtiers de développement, personnages qui relient et mettent en communication des mondes, des systèmes de sens, des cultures différentes" (Blundo 1995 :77). susceptibles d'en profiter le plus. Tout de même, c'est aussi le groupe le plus délicat lorsqu'il s'agit de rapprocher les différents systèmes de pensée :

A : A chaque activité, il faut d'abord prendre contact avec leur mari, expliquer le contenu du message. Après, eux, ils retournent chez eux déjà, parlent à leurs femmes, et une fois que leur réunion est convoquée, une réunion concerne uniquement les femmes, là, il n'y a pas de problème, les femmes viendront en grand nombre parce qu'elles ont déjà envie de parler (...)

$\mathrm{M}$ : Ah bon. Parce que ce j'ai remarqué aussi, c'est que bon s'il y a des réunions, les ateliers avec les femmes, il n'y a aucune femme qui parle. Les hommes parlent entre eux, ce sont les femmes qui sont à côté (...) on ne parle pas avec une femme.

A : Oui, bien, je suis en train de débattre ce problème parce que c'est maintenant une grosse erreur (...). Dans la prise de conscience pour des femmes, il faut que la femme soit complètement responsable de ce qu'elle attend, de ses besoins et de ce qu'elle ne veut pas. Elle a le plein devoir d'exprimer ses besoins.

A : Donc, si par exemple à chaque fois qu'un problème se pose, on s'adresse à leurs maris pour que eux, ils trouvent les solutions, je crois ça ne fait pas la promotion. C'est pas possible.

31 Au cours de l'entretien, N.A. met en relief le succès de son travail de sensibilisation, ce qui permet à certaines femmes de prendre quelques décisions de manière plus autonome. Grâce à une formation à la conservation de tomates par exemple, les hommes ont pris confiance en N.A, ayant vu que le nouveau savoir acquis par les femmes était utile pour l'ensemble de la population. Dans l'ensemble, elle décrit différemment la situation des femmes au début et à la fin de l'entretien. D'abord, elle parle de gros problèmes de résistance contre la promotion féminine du côté des 
hommes et des marabouts. Or, vers la fin, elle souligne de plus en plus sa position d'intermédiaire couronnée de succès : "La promotion féminine est en train de faire de grands pas!" Ceci peut être lié au caractère officiel de l'entretien résultant de l'utilisation d'un magnétophone. A d'autres occasions, par exemple sur le chemin vers un village, N.A. me donnait d'autres renseignements que lors de la présentation dans le village. Son langage différait aussi au moment où nous nous rendîmes au siège de l'ADAK (Association des Agriculteurs de la région de Kaolack), à Boulel, afin de discuter avec la présidente du groupement des femmes et avec des "facilitateurs" de l'organisation paysanne. N.A. est en bon contact avec les personnes impliquées dans l'organisation paysanne ainsi qu'avec les personnes liées aux bailleurs de fonds. Ceci se fait par exemple par l'intermédiaire d'une Française résidante à Kaffrine depuis plus de vingt ans. D'habitude, nous avions plutôt un contact informel, ce qui s'exprime par le tutoiement. Néanmoins, pendant que le magnétophone était en marche, elle me vouvoyait.

N.A. figure comme une dirigeante régionale au niveau de l'arrondissement de Kaffrine. Grâce à ses contacts multiples aux villages, elle arrive en général à présenter un 'village modèle' correspondant à l'intérêt spécifique du visiteur, en particulier quand il s'agit d'un représentant d'un bailleur de fonds étranger. Comme je lui avais expliqué que les confréries islamiques étaient au centre de l'intérêt de ma recherche, N.A. m'a aussitôt emmené dans un village "très islamisé" d'après elle. De plus, comme elle savait que je m'intéresse particulièrement aux femmes et que j'étais en contact avec un projet de la coopération technique allemande (GTZ), elle a pris beaucoup de temps à me présenter le projet de la Banque Mondiale (mentionné ci-dessus) en cours d'évaluation dans ce village. Une partie de ce projet consiste à établir des classes d'alphabétisation en la langue régionale wolof, en majorité pour les femmes. Lors de notre première visite du village de Médina K., elle tint alors un long discours en salle de cours sur le succès de ce projet et sur la motivation des femmes. A l'occasion d'un deuxième séjour au village sans avoir été accompagnée par N.A., je me rendais compte du fait que la motivation n'était pas si forte que cela dans la vie quotidienne, mais que le village était tout de même un exemple qui dépassait la moyenne au niveau des conditions de vie. Les études de village présentées ci-dessous sont susceptibles d'illustrer l'interpénétration de règles islamiques et d'autorités religieuses avec les modes d'organisation des nouveaux groupements paysans à l'échelle villageoise sans que ces tendances déstabilisent le fonctionnement de l'Etat (cf. Villalon 1995).

Etude de village à Medina $\mathrm{K}$.

Le groupement de Médina K. qui existe depuis 1985 entreprend trois activités principales : culture d'un champ collectif d'arachide, élevage du bétail, petit commerce. Ce groupement est enregistré sous forme de GIE (Groupement d'Intérêt Economique). Dans le cadre du conseil agricole de l'Etat subventionné par la Banque Mondiale, la FONGS (Fédération des Organisations Non-Gouvernementales du Sénégal), en tant qu'exécutant, a développé un projet d'alphabétisation qui se trouve actuellement dans sa phase d'évaluation. Depuis 1994, chaque membre de l'organisation paysanne obtient un crédit de $35 \mathrm{~kg}$ de mil du grenier commun (une maison en pierre couverte de zinc) afin d'assurer la survie pendant l'hivernage, jusqu'à la prochaine récolte. La première année, le crédit fut accordé au mois d'août. Chaque femme de l'organisation reçut un crédit de 3000 FCFA ( 30 FF) afin de l'investir dans l'achat d'une chèvre, d'un mouton ou d'arachide (pour la production de l'huile pour le marché). En collaboration avec un 
bailleur de fond néerlandais, l'organisation paysanne creusa un puits d'une profondeur considérable.

Le problème majeur, surtout en dehors de la période d'hivernage, réside dans le manque d'eau à cause des puits taris. Comme les femmes sont chargées de s'occuper des tâches ménagères, en particulier de la préparation des repas, elles sont surtout concernées par l'obligations de satisfaire aux besoins élémentaires. A cause du déboisement résultant de la production commerciale de charbon de bois et des besoins de bois de chauffage, l'approvisionnement en bois pour faire la cuisine devient de plus en plus difficile et requiert de plus en plus de temps.

Une particularité du quartier (partie du village dans laquelle habite une grande famille) de Médina K. dans lequel j'habitais était le fait que tous les hommes, à part trois, vivaient en Italie.

Régulièrement, les femmes recevaient des coups de téléphone, du courrier et au moins une fois par an la visite de leurs maris de Naples. En conséquence, les femmes étaient seules chargées de la culture des champs, des tâches ménagères et de l'éducation des enfants. La régularité avec laquelle les hommes, vivant en Italie et unis par l'appartenance à la confrérie des Tidjanes, rendent visite à leur village d'origine diffère considérablement. S.K., mon hôte, avait déjà eu deux fois durant cette année la visite de son mari. D'autres femmes du village parlent de plusieurs années d'intervalle entre les visites de leurs maris. Sur les photos reçues de leurs maris, on voyait les hommes du même village ensemble, souvent dans un logement dans lequel divers objets destinés au petit commerce (cassettes, stylos en plastique, porte-clés, bijoux etc.) étaient empilés sur des étagères. C'est pour cela que les femmes reçoivent régulièrement des colis contenant des petits objets tels que des bijoux, de la vaisselle, des souvenirs, des articles de toilette etc. Ces objets, portant des inscriptions en italien ou en français, étaient entassés dans un placard vitré. Sur d'autres photos on pouvait voir en plus des Sénégalais venant d'autres villages ou des Italiens et des Italiennes. De très grands portraits du mari étaient accrochés au mur, à côté de calendriers et d'images de marabouts. Quelques unes des photos ainsi que des papiers témoignaient d'un ou plusieurs séjours en France. Dans son enquête, Victoria Ebin (1993, in: Grégoire, Labazée : 117) parle des liens étroits entre les membres de confréries islamiques à l'étranger. La veille d'un baptême, le père de l'enfant rendit visite à sa famille et se rendit dans le quartier voisin pour distribuer du courrier et pour donner des nouvelles aux femmes dont le mari était resté en Italie. Dans ce quartier, trois frères de même père et de même mère ont émigré vers Naples. Le mari de $\mathrm{K}$. travaille dans une usine de tracteurs et dans des fermes où il s'occupe également de tracteurs. Les autres hommes font du petit commerce.

D'après Victoria Ebin ${ }^{2}$, l'implication dans le secteur informel est typique pour des Mourides émigrés. Quoiqu'il s'agisse à Médina K. de membres de la confrérie des Tidjanes dont les réseaux économiques sont moins pris en considération dans la littérature que les activités commerciales des Mourides, certains aspects mentionnés par Ebin concernant des Mourides sont transférables aux frères décrits ci-dessus. Dans son étude sur Dakar, Roman Loimaier ${ }^{3}$ se réfère avant tout aux Mourides, mais mentionne des structures semblables chez les Tidjanes: "Les réseaux des Mourides, comme ceux de la Tigânîya, dépassent toute frontière sociale ou économique existante, sans s'occuper des modèles de couches sociologiques ou des théories de développement...Le fait de relier au réseau des secteurs ruraux et urbains, 'traditionnels' et 'modernes', la présence de 
groupements sociaux les plus variés ayant un lien de clientèle et de soumission à l'autorité religieuse d'un marabout, explique le succès particulier des réseaux religieux sur le plan politique et économique..." (Loimaier 1994: 257, traduit par Mo.S.). En somme, le niveau de vie des femmes du village est largement au-dessus de la moyenne en milieu rural, où la possession d'une télévision p.ex. est très rare. Pendant une pré-conférence au 23ème congrès mondial en sociologie (qui se déroula en juin 1994 à l'Université de Bielefeld), Abdou Salam Fall de l'Institut Fondamental d'Afrique Noire à Dakar montra des exemples pour l'influence des marabouts en milieu urbain. D'après lui, les représentants de la politique officielle sont obligés de passer par des marabouts afin que certaines mesures politiques soient appliquées par la population dakaroise. "L'élite maraboutique flirte avec l'élite politique, puis s'allie à elle...Par ces alliances, l'Etat peut toucher l'ensemble des populations. Il peut contenir toutes velléités revendicatives des populations et, ce faisant, gérer le compromis social" (Fall 1994: 300). Un exemple pour ce compromis toléré est le contrôle du marché dakarois Sandaga par les Mourides.

Selon K. (un membre du groupement féminin), les hommes de Médina K. ont émigré sous directive de leur marabout. A l'arrivée, ils furent hébergés dans un appartement appartenant à d'autres membres de la communauté du même marabout. Ebin (1993: 121) écrit que les confréries établissent des communautés fortement soudées et procurent à ses membres les moyens de déplacement. A l'étranger, la communauté sert également à ses membres de réseau de sécurité sociale, et elle leur offre de ce fait une allocation de chômage, un approvisionnement en médicaments, une assurance vie, elle s'occupe de l'éducation des enfants et prend soin des femmes (Ebin 1993: 115). Ces dahiras (de l'arabe dâ'ira : cercle, domaine, zone) nées à l'étranger suivent l'exemple des organisations urbaines de l'intérieur du pays, dont le but était d'ailleurs d'établir un lien stable entre les communautés urbaines des Mourides et les leaders de la confrérie Sufi en milieu rural (cf. Diop 1980).

Sur le plan religieux, à Médina K., un Imam dirige une école coranique qui ne fonctionne pas en ce moment. Cette école située en plein air et entourée d'une clôture en paille en forme de spirale, se situe à l'arrière d'un bâtiment en pierre, destinée à accueillir une classe d'alphabétisation, pour adultes, en wolof. Alors qu'on cultive habituellement dans la plupart des villages un champ pour la mosquée qui est surveillé par l'Imam du village, à Médina $\mathrm{K}$. on en cultive même deux pour des raisons religieuses: un pour le grand marabout Abdou Aziz Sy de la confrérie des Tidjanes à Tivavouane avec lequel la population du village est en bonne relation et un autre pour Moustapha Sy, leader du mouvement des Moustarchédines. Abdou Aziz Sy est le Khalif(chef actuel de la confrérie) et grand-père de la famille Sy qui a introduit la confrérie des. Tidjanes au Sénégal: "Mais c'est El Hadj Malick Sy qui a introduit le Tidjanisme en pays wolof par son enseignement et par sa conduite exemplaire. D'origine toucouleur, il naquit vers 1855 : au village de Gaya près de Dagana, dans un Walo intellectuellement influencé par la Mauritanie arabe" (Marone 1970 : 148). Ainsi s'explique aussi l'influence arabe sur les Tidjanes, au contraire de l'enracinement des Mourides en Afrique noire.

Depuis la fondation du mouvement Moustarchédine, il y a 14 ans, les talibés, hommes et femmes, cultivent un deuxième champ dans le village pour leur leader Moustapha Sy D'après d'autres sources, ce marabout lié au village de Médina K., voulait s'emparer du pouvoir et diviser Tivavouane. Les activités subversives de Moustapha Sy sont 
confirmées par des articles de journaux traitant de la participation de Moustarchédines aux protestations du 16 février 1994 et à l'attentat contre l'avocat Babacar Seye le 15 mai 1993. Pendant tout notre séjour, ce sujet était à la une dans tous les journaux, car le procès contre les auteurs de l'attentat venait de débuter. C'est dans ce contexte qu'on a relevé aussi des connivences avec le parti de l'opposition, le PDS (parti Démocratique Sénégalais).

41 Les habitants de Médina K. semblaient être au courant de ces reproches. Les femmes n'étaient pas sûres de la libération de Moustapha Sy qui, comme le président du PDS Abdoulaye Wade, avait été emprisonné à la suite des révoltes du mois de février 1994. J'eus l'occasion d'assister à la première visite de la sœur du marabout emprisonné. Elle arriva au village sur une charrette couverte de velours, accompagnée de plusieurs femmes et d'un homme, dans le but de convoquer l'assemblée du village pendant laquelle elle s'adressa à la population au nom de son frère et demanda leur soutien. En outre, elle énuméra quelques règles de conduite à respecter, p.ex. que l'homme doit bien traiter sa femme car dans le cas contraire elle risquerait de quitter son mari. Il paraît que les habitants du village reçoivent régulièrement des ordres de la part de Moustapha Sy. Une des directives concerne toujours la participation au Gamou, la grande fête commémorative de l'anniversaire du prophète. Pendant les dernières années, le village avait été interdit de participation à la fête, car Moustapha Sy voulait boycotter la cérémonie. Ceci fut encore le cas en 1994. Le boycott de la célébration par Abdoulaye Wade semble être lié à cet ordre : "A Tivavouane en tout cas le "faux bond" des libéraux lors de la cérémonie officielle serait lié, pour plusieurs pèlerins, à l'affaire Moustapha Sy, le leader des Moustarchédines actuellement en prison et allié de taille de $\mathrm{M}$. Wade et de son parti. On sait également que les Moustarchédines eux-mêmes ont 'boycotté' la cérémonie de la ville sainte, préférant célébrer la naissance du prophète autrement" (Sud quotidien 22.8.94:5). De plus, le rapport entre la vénération de Moustapha Sy de la pan des habitants du village et le comportement électoral est évident. Trois cinquième de la population votent pour le PDS ; ceci est confirmé par de nombreuses affiches d'Abdoulaye Wade accrochées dans les cases.

Jusqu'à l'interdiction du mouvement des Moustarchédines en février 1994, une daara aux traits fondamentalistes a existé à Médina K.. Par l'intermédiaire de la radio, des ordres avaient été transmis, notamment l'interdiction de danser en public. La conséquence fut qu'on avait supprimé même les tamtams (danse sur la musique de tambours) au village. Deux fois par semaine, des rassemblements avaient lieu, ayant pour but d'apprendre les obligations religieuses et de pratiquer ensemble la religion. A cela s'étaient ajoutés des chants récitatifs et des prières. Après l'interdiction du mouvement, les danses spontanées recommencèrent et l'écoute de musique fut autorisée à nouveau. Même s'il n'y avait plus de rassemblements au moment où je fis ma recherche, on sentait nettement l'influence du mouvement des Moustarchédines et les liens avec la famille du jeune Moustapha Sy.

Etude de village à Medina $\mathrm{G}$.

43 A Médina G., les activités du groupement mixte comprennent la culture d'un champ collectif de mil et d'arachide, l'entretien d'un moulin à mil et l'élevage de bœufs. Pendant l'hivernage, le stock de mil du grenier commun du groupement villageois est distribué sous forme de crédit entre les membres de l'organisation. Avec la participation de tous les membres, un nouveau puits fut construit, afin de palier au manque croissant d'eau. Les femmes de l'organisation paysanne, regroupées comme les 
hommes en 1983, obtinrent toutes une chèvre pour l'élevage. Le bâtiment de la classe d'alphabétisation (en langue régionale wolof) construit en 1991 était tombé en ruines, de sorte qu'en ce moment, il n'y avait aucune possibilité de formation pour les adultes. Ce sont surtout les femmes qui en pâtissent car elles constituaient la grande majorité des élèves. La présidente du groupement non-mixte qui avait suivi une formation comme assistante de santé dans le cadre de la promotion féminine, organisa une campagne de nivaquinisation contre le paludisme et mit en place plusieurs mesures d'hygiène, ce qui fut réalisé avec l'aide financière du groupe de femmes et du groupement paysan mixte.

Contrairement à la situation à Médina K., la plupart des hommes de Médina G. était présent. Une partie des jeunes hommes célibataires quittent le village pendant la saison sèche pour aller travailler en ville. Ce sont cependant les femmes et les jeunes filles qui s'occupent essentiellement de la nourriture. Comme les habitants du village ne résident en ces lieux que depuis 50 ans, beaucoup d'arbres poussent encore à proximité du village, ce qui rend la collecte de bois plus facile. Mais cette situation géographique fait que, pendant la saison sèche, des habitants du village s'adonnent à la production de charbon de bois, qui est ensuite vendu à bas prix aux marchands qui à leur tour le revendent en ville.

Tant que les puits ne sont pas taris, les femmes vont chercher l'eau. Depuis trois ans, il existe un nouveau puits, qui fut financé par les membres du groupement paysan et les autres habitants du village. il se trouve à environ dix minutes à pied. La répartition du travail, dont les femmes portent la charge, est un obstacle à la mise en place d'un projet d'alphabétisation à Médina G., car elle ne leur laisse pas le temps de suivre des cours à l'école. L'installation autonome de machines (moulins à mil, décortiqueuses) est très difficile pour les habitants du village, car il faut qu'ils fournissent eux-mêmes 15 à 50\% du capital nécessaire pour avoir ainsi droit à un crédit

De grands problèmes se posent lors de l'approvisionnement en semences. D'après les paysans, les institutions de l'Etat réclament des payements pour des semences qu'ils n'ont jamais obtenues. On soupçonne les fonctionnaires au niveau de la communauté rurale de les avoir détournées, au lieu de distribuer les marchandises de l'Etat. Gudrun Lachenmann (1991 :78, traduit par Mo.S.) écrit à propos de la tension latente entre le désengagement de l'Etat et la responsabilisation des paysans: "La politique du désengagement de l'Etat n'est pas vraiment perçue comment libérateur, mais plutôt comme une menace et un danger de voir l'Etat s'approprier leurs institutions et leurs ressources. Les structures étatiques restantes sont tellement affaiblies (surtout par les mesures de compression budgétaire imposées de l'extérieur) qu'elles ne jouent plus un rôle qui encourage le développement, mais réagissent nécessairement avec jalousie (surtout vis-à-vis de moyens venant de l'extérieur) et bloquent certains processus. D'une part il s'agit donc d'une libéralisation et diminution de services étatiques d'encadrement, de subventions etc., et d'autre part de plus en plus d'ingérence de l'Etat. Cela va si loin que les organisations paysannes ne se demandent pas seulement si elles peuvent mais encore comment remplir des fonctions économiques et d'autres (telles que commercialisation, ravitaillement en semences, régime des crédits, formation) pendant la crise, comment elles peuvent préserver de bonnes relations avec l'administration et le système politique, tout en étant obligées de créer des possibilités de ravitaillement pour des conseillers ruraux de l'Etat licenciés etc.". W.N., un animateur du groupement paysan, était présent pendant une période de mon séjour au 
village. Avant de participer aux stages de formation de la FONGS dans le but de devenir animateur, il avait remplacé son oncle au poste de peseur dans un dépôt d'arachide à Keur Yoro, le village voisin. Avec sa nouvelle tâche, il est donc passé des structures étatiques aux structures non-étatiques. Il est vrai qu'il n'a que pendant les vacances de la FONGS, pendant l'hivernage, l'occasion de s'occuper de son champ d'arachide, envahi par beaucoup de mauvaises herbes entretemps. De plus, ses plantes d'arachide étaient plus petites que sur les champs aux alentours; de là on peut donc induire qu'il n'avait pu semer qu'à une époque défavorable.

ce qui concerne le processus de décision, les habitants du village s'adressent toujours au marabout ou à l'Imam (les autorités religieuses du village) pour demander des conseils en accord avec la religion. Si un projet va dans un sens contraire à la religion selon ces autorités, sa réalisation entraîne de grosses difficultés. A Médina K., l'installation d'une classe d'alphabétisation fut soutenue par toutes les autorités du village, de sorte que les femmes se sentaient fort encouragées à suivre les cours. Néanmoins, l'Imam de Médina G. n'était pas vraiment convaincu de la justesse de ces propositions, car, selon lui, les femmes auraient d'autres occupations plus importantes, les empêchant d'aller à l'école ${ }^{4}$ :

"D'habitude, les femmes ne respectent pas la religion; les hommes sont plus compétents. Quant aux femmes, ce n'est pas un problème de temps. Il y a des moments pendant lesquels les femmes ont le temps, mais elles ne veulent pas apprendre. Il y a des femmes qui veulent, mais elles n'ont pas les moyens parce qu'elles n'ont pas de marabout qui leur apprend quelque chose. A cause des femmes, il y a un retard car elles n'ont rien appris. Dans la religion musulmane, l'homme doit savoir et la femme aussi doit savoir, mais l'homme a déjà acquis ses connaissances. La femme n'a rien compris et cela entraîne un retard. La religion musulmane dit que l'homme doit venir d'abord. C'est toujours l'homme qui doit parler. Les femmes doivent toujours être soumises. Nous ne sommes pas contre la promotion féminine. Mais les femmes font des choses au sein $d u$ groupement que la religion ne recommande pas. Je n'ai pas peur que la promotion féminine s'installe, mais l'islam ne le veut pas".

Par conséquent, les femmes se trouvent coincées entre leurs intérêts et des obstacles qui sont souvent justifiés par la religion. Des interprétations multiples basées sur la religion font que certains faits sont appréciés d'une manière différente.

50 Avant d'entreprendre l'étude de terrain, je partais de l'idée que les fonctions qui autrefois avaient été accomplies par les confréries islamiques se trouvaient désormais dans les mains de l'organisation paysanne. Ceci est confirmé en partie du point de vue empirique lorsqu'on considère les champs collectifs. A Médina G., les revenus du champ cultivé pour la mosquée furent utilisés pour des besoins de la communauté villageoise, p.ex. pour la sécurité sociale des pauvres. Depuis l'installation d'un champ collectif de l'organisation paysanne, les aides financières proviennent du produit de la récolte. Le champ pour la mosquée existe toujours et sert maintenant à financer la construction d'une nouvelle mosquée.

51 Faute de place, il n'est pas possible d'énumérer ici les buts spécifiques des Programmes d'Ajustement Structurel soutenus par le Fonds Monétaire International (FMI) et la Banque Mondiale, mais il est nécessaire de regarder brièvement la situation actuelle concernant les groupements paysans. Comme la plupart des mesures ont échoué (cf. Duruflé 1988: 84), un nouveau programme de secteur pour l'agriculture devait être formulé, et la FONGS a réussi à se faire inviter aux négociations. Le Comité National de 
Concertation des Ruraux (C.N.C.R.) fut créé afin de servir d'interlocuteur pour ces négociations. Dans la Déclaration de Thiès, on met l'accent sur la nécessité de participation de la population rurale aux programmes de développement: "Cette démarche vise, entre autres objectifs, à doter les ruraux et leurs organisations, des moyens d'améliorer leur compréhension des politiques de développement rural, afin d'opérer les choix les plus judicieux et de mettre en œuvre les actions les plus pertinentes, eu égard à l'émergence de la Société Civile comme nouvelle donne de la démocratie sénégalaise" (République du Sénégal, C.N.C.R., 1993 : 2). Il en découle que depuis 1990, des représentants de la FONGS sont au moins consultés pour des décisions de politique agricole, un fait qui résulte de la nouvelle philosophie de la Banque Mondiale de coopérer avec les ONG, et de ce fait celles-ci jouent un rôle de plus en plus important de conseiller (Cissokho 1994 : 47).

Les confréries islamiques en tant que vecteurs de mutations sociales Du village à la ville, de Dakar à Marseille - origines de la daara

Les organisations urbaines des dahiras qui, à partir de Dakar, se sont développées en tant que réseaux mondiaux, trouvent leur origine dans des unités de production rurales, appelées daara (dérivé de l'arabe dâr : maison, ferme). Chaque marabout préside une ou plusieurs communautés dont la tâche consiste à étendre constamment la gloire de leur maitre (cf. Magassouba 1985: 35). Ceci se passe d'une part par le travail non-rémunéré dans les champs du marabout, et d'autre part, par la récitation de versets $\mathrm{du}$ Coran une ou deux fois par semaine et la répétition dans un état de transe de chants religieux. Les jeunes élèves coraniques du marabout appartiennent aussi à la daara. A Médina G., les garçons travaillent tous les jours de huit heures à midi et de 14 à 18 heures sur le champ du marabout. Tous les vendredis, les hommes du village appartenant à la daara travaillent sous surveillance de l'Imam sur le champ de la mosquée. Jusqu'à la création de l'organisation paysanne à Médina G. en 1985, les revenus de la récolte furent utilisés pour subvenir aux besoins collectifs, p.ex. pour creuser un puits.

Entretemps, ce champ si soigneusement cultivé existe toujours, mais le produit de la récolte est réservé à la construction d'une nouvelle mosquée. Cet exemple illustre bien que la création du groupement paysan a entrânée un déplacement de la responsabilité. En complément au champ pour la mosquée, un champ collectif de mil et un champ d'arachide sont cultivés pour le groupement paysan. On remarque beaucoup plus de mauvaises herbes sur ces champs-là, ce qui révèle d'une culture un peu plus négligée, mais comme ils sont plus grands, la récolte y est également plus importante.

Dans l'étude du village de Médina K., on voyait qu'il y a là aussi une daara de la confrérie des Tidjanes dont les membres cultivent le champ de leur marabout. Comme décrit plus haut, les marabouts agissent dans ces cas en tant qu'intermédiaires entre le niveau gouvernemental et la population. Cette stratégie est la conséquence d'une structure dont la France en tant que puissance coloniale s'était déjà servie :

"Quelle place les marabouts occupent-t-ils dans le nouveau système ? Si l'on considère qu'ils contrôlaient eux-aussi les communautés villageoises et que le développement de leur entreprise sur une partie importante de la société sénégalaise a suivi l'expansion coloniale, plusieurs réponses peuvent être formulées. Intéressés par l'arachide, les marabouts comme les paysans sont intégrés dans l'économie de traite; moins discrédités que les anciennes familles aristocratiques, ils sont plus écoutés par les paysans. De même, ils sont plus aptes à fournir un appui idéologique au colonisateur 
pour légitimer sa domination. Leur pouvoir, dans les communautés paysannes est, de par sa nature, différent de celui des chefs de cantons ou de villages" (Diop, Diouf 1992 : 70).

Comme le relèvent certains articles de journaux ${ }^{5}$, les liens ne sont pas seulement intenses entre le gouvernement et les confréries, mais aussi entre les partis de l'opposition et les confréries :

"Le fait est réel, Moustapha Sy draine des milliers de partisans acquis à la mystique entretenue de la mémoire de Maodo Malick Sy. Récoltant les dividendes d'un charisme atavique, le petit-fils Moustapha appuie sur la pédale de la terreur sacrée que les marabouts inspirent aux hommes politiques. Pourtant Wade comme Diouf savent parfaitement que l'activisme politique du jeune marabout est définitivement frappé de suspicion, parce que inscrit dans une longue tradition de relations féodales tropicalisés" (promotion 26.9.94).

Dans le même article, on prétend que déjà le premier président Senghor avait non seulement remporté l'élection présidentielle à l'indépendance en 1960, mais aussi son siège à l'Assemblée Française en 1951 en partie grâce aux marabouts. Les liens entre les confréries, les organisations paysannes et les partis existant à travers toutes les couches sociales ne sont pas toujours clairs. Pour la région de Fatick (voisine de la région de Kaolack présentée ci-dessus), Léonardo A. Villalon (1995) a en outre démontré les gestes et objets symboliques représentant les liens entre l'Etat et le pouvoir religieux, p.ex. les visites mutuelles de courtoisie à l'aide matérielle (haut-parleurs etc.) de la part du gouvernement lors des cérémonies religieuses. Pendant notre enquête, nous avons non seulement trouvé des exemples pour ces liens, mais aussi connu de nombreux personnages qui lient leurs convictions religieuses à leurs activités pour les organisations non-gouvernementales, comme la coordinatrice régionale de la FONGS pour la promotion féminine, N.A., qui, quant à elle, est une Mouride pratiquante et en même temps militante convaincu du PDS, et elle le montre ouvertement. Avec ses contacts multiples (entre autres aux bailleurs de fonds internationaux), elle fait partie du groupe des courtiers du développement défini par Jean-Pierre Olivier de Sardan et Thomas Bierschenk (1993 : 71) : "Par 'courtiers locaux de développement', nous entendons les acteurs sociaux implantés dans une arène locale qui servent d'intermédiaires pour drainer (vers l'espace social correspondant à cette arène) des ressources extérieures relevant de ce que l'on appelle communément "l'aide au gouvernement"'. Giorgio Blundo (1995: 95) se demande déjà si ces acteurs sont susceptibles de prendre la place que les représentants religieux avaient occupé pendant l'époque coloniale :

"Peut-être est-ce le tour des responsables paysans et de leurs organisations d'assurer cette position?" Au lieu d'un changement d'acteurs, l'interpénétration entre les différents réseaux sociaux, politiques et économiques continuera à mon avis sans exclure le champ religieux qui, quant à lui, est superposé aux autres domaines (cf. Salzbrunn $1994:$ :461).

Battement de tambour à la pleine lune - la dahira des Baye Fall à Koungheul

Vers la fin de mon séjour, je rendis visite à A.N., une animatrice volontaire de la Banque Populaire à Koungheul (actuellement fermée faute de fonds), qui avait été formée dans le cadre du programme d'alphabétisation pour adultes de la FaNGS. Célibataire, elle dispose d'une formation interrompue de premier cycle et vivant en ce moment avec sa fille dans la maison parentale à Koungheul. En plus de cette fille de trois ans, elle a trois 
autres enfants de deux pères différents, qui vivent avec la mère de A.N. en Casamance, dans le sud du Sénégal. En tant qu'animatrice, elle organise des formations dans les villages voisins. En outre, en tant que Mouride convaincue, elle appartient à une dahira des Baye Fall. Cette branche des Mourides se réfère au Cheikh Ibra Fall, l'élève préféré de Sérigne Touba. Comme les Baye Fall sont dispensés des obligations de prier et de jeûner grâce à la fidélité de leur fondateur Ibra Fall vis-à-vis du père des Mourides Cheikh Ahmadou Bamba, et que la plupart d'entre eux se nourrissent grâce à des aumônes, en général, ils ne sont pas très bien vus par la population. Un jour que j'étais assise dans la cour d'un village avec ma traductrice N.D. (qui appartient aux Tidjanes, mais sans être pratiquante pour autant), un Baye Fall passa pour demander l'aumône. N.D. le gronda et le traita de fainéant. De même, lorsqu'un jour un Baye Fall entra dans un restaurant à Kaolack géré par une Chrétienne, le personnel n'était pas content, à cause de la réputation de malhonnêteté, non prouvée, de ce Baye Fall. Moriba Magassouba (1985:35) écrit à ce propos qu'on supposait chez les Baye Fall un manque de respect de la loi, étant donné les libertés qu'ils prenaient sur le plan religieux.

Néanmoins, les Baye Fall se font remarquer par leur disponibilité illimitée vis-à-vis de leur Khalife, d'où leur appellation de soldats des Mourides. J'ai pu assister à un rassemblement nocturne de la dahira de A.N., et observer le comportement dévoué des membres envers le président de la lùlhira. A la pleine lune, les hommes et les femmes s'exerçaient à la récitation de vers coraniques, récitation qui se transformait peu à peu en chants à haute voix. Au bout de trois heures, les Baye Fall, se balançant au rythme d'un tambour, atteignirent une sorte d'extase et tournèrent en rond en chancelant le regard comme transfiguré. La conviction religieuse des Baye Fall ne s'exprime pas seulement par les rassemblements qui ont lieu deux fois par semaine, mais aussi par leur opinion concernant la relation des talibés avec leur marabout. Lorsque je voulus m'informer d'une manière critique sur la répartition du pouvoir social et économique, je n'obtins pas de réponses mettant en question les structures existantes. Mais il est vrai que les organisations paysannes en tant que nouvelle force dynamique étaient appréciées et notamment soutenues, p.ex. par le travail pratique réalisé par A.N. Elle ne voyait en aucun cas un rapport de concurrence entre les confréries et les groupements paysans. Selon A.N., les organisations paysannes facilitaient de manière considérable la vie quotidienne des femmes, en s'engageant pour des améliorations concrètes. Au centre de la vie de A.N., on pouvait déceler la croyance en Dieu et sa vénération. Cette vénération se manifeste concrètement dans son admiration pour le fondateur des Mourides, Cheikh Ahmadou Bamba, dont elle avait un portrait géant peint sur le mur de sa case au-dessus de son lit, également pour le Cheikh Ibra Fall, dont elle répète sans cesse le nom dans ses chants.

Le vendredi, on cultive le champ de la mosquée. L'importance de la religion dans la vie quotidienne

Beaucoup d'activités de la vie quotidienne sont imprégnées par les principes coraniques ou par des citations de savants musulmans. En ce qui concerne l'attitude vis-à-vis du travail, les Mourides se réfèrent à une citation importante de Cheikh Ahmadou Bamba (in: Mawaahibu Naafih): "Travaille comme si tu ne devais jamais mourir, prie Dieu comme si tu devais mourir demain". Dans la pratique, ce dogme connaît des effets négatifs extrêmes: certains talibés se tuent littéralement en travaillant sur les champs de leur marabout, à tel point que Moriba Magassouba (1985: 
35) parle d'esclavage et de sacrifice suprême. Mais il faut dire que la discipline et les contraintes varient beaucoup d'une dahira à l'autre selon le caractère du marabout. L'appréciation de ces situations diffère également selon les auteurs. Victoria Ebin (1993 : 114), quant à elle, met en relief l'utilité et la protection que cela procure aux membres d'une confrérie: "On dit souvent que les relations du cheikh avec les talibés relèvent de l'exploitation. Des recherches effectuées auprès des agriculteurs Mourides montrent, au contraire, que ceux-ci ne travaillent pas vraiment plus durement que les autres Sénégalais, et qu'ils ne subissent pas de réelle pression pour effectuer des dons à leurs marabouts". Copans (1988: pp.154) qualifie la valeur du travail de chaque talibé plus symbolique et idéologique que technique et économique. Selon lui, l'exploitation des talibés par les marabouts se dégage cependant lorsqu'on regarde le système dans son ensemble. Les travaux communs en tant qu'institution sont la base d'un système d'exploitation.

L'obligation au travail des membres des confréries ne ressort pas seulement au niveau de la disponibilité pour le marabout, mais aussi au niveau de l'engagement pour leurs propres champs. A Médina G., un village possédant une communauté tidjane très vive, les membres du groupement paysan s'occupent très soigneusement de leurs propres champs ainsi que de ceux du groupement villageois. Cela a pour conséquence que la banque à mil commune servant à procurer du mil sur crédit fonctionne de façon excellente dans ce village. A Médina K., un village fort islamisé, le système de distribution de mil sur crédit en provenance du grenier commun construit par les membres du groupement villageois fonctionne également depuis plusieurs années. Prenant en compte la situation générale du pays, cela paraît particulièrement positif. On pourrait donc en conclure que la pratique intense de la religion est positive pour les activités de l'organisation paysanne. Pour approfondir cette analyse, il nous faudrait faire des études comparatives dans des organisations paysannes dont les membres sont moins engagés sur le plan religieux que ceux décrits ci-dessus. Mais il est vrai que les organisations paysannes soulignent toujours que leur action correspond aux valeurs de l'islam, tout en affirmant qu'elles ne se considèrent pas comme institutions religieuses : "La FONGS est une organisation laïque" (Cissokho, Interview et Conférence à l'Université de Bielefeld, 1994). Néanmoins, les membres fondateurs d'un groupement paysan appartenant à la FONGS se sont posés la question de savoir comment ils pouvaient appliquer à l'économie monétaire des règles qu'ils considèrent comme islamiques. Eva-Maria Bruchhaus (1989), après avoir assisté à certains réunions du groupement, énumère les stratégies alternatives, comme un système de crédit de mil distribué en juin et remboursé en espèces par les paysans après la vente de la récolte de l'arachide entre les mois de janvier et mars.

Sur le plan de la pratique concrète de la religion, la prière occupe une place importante dans l'emploi du temps. Pendant mon séjour à Médina G., tous les hommes présents ont suivi très sérieusement les cinq prières de la journée (à l'aube, à midi, l'après-midi, au coucher du soleil et le soir). Comme d'habitude, ils déroulaient une natte de prière sous le toit en paille au milieu de la ferme. Par contre, j'ai rarement remarqué des femmes priant en public. Dans les villages, on prétendait que les femmes priaient chez elles, à la maison. Quelques hommes étaient cependant de l'avis que les femmes négligeaient leurs devoirs de prière. N.A., la coordinatrice de la FONGS pour la promotion féminine, priait d'habitude dans sa chambre en présence d'autres personnes. Quand nous étions en voyage, elle avait soin de rattraper des prières omises. Lors de mon départ de 
Médina G., nous priâmes ensemble avec tous les hommes, femmes et adolescents présents et nous reçûmes la bénédiction de l'Imam.

A Médina K., les femmes racontaient qu'un voyage à la Mecque était l'objectif primordial dans leur vie et elles faisaient des économies dans ce but. Comme quelques hommes gagnent plus d'argent en Italie que dans leur patrie, cet espoir est en effet réaliste. Une femme raconta que son projet de voyage dut être annulé à la dernière minute car une somme importante d'argent lui avait été volée. Un après-midi, une femme m'apporta un View-Master, une sorte de projecteur mécanique de diapositives en miniature au moyen duquel on pouvait visionner des images de La Mecque. Le disque introduit dans l'appareil portait des descriptions en anglais et provenait d'Arabie Saoudite.

A Médina G., les revenus ne suffissent pas pour entreprendre un pèlerinage. Les célébrations plus locales prennent alors une place d'autant plus importante. Comme j'étais présente dans la semaine qui précédait le Gamou, j'ai pu observer que presque toutes les activités, surtout celles des femmes, étaient concentrées sur la préparation de cette fête qui devait avoir lieu dans le village voisin. Le Gamou est la commémoration de l'anniversaire du prophète Mohammed, et est fêté principalement par les Tidjanes. Les croyants se rassemblent à la Mosquée, écoutent jusqu'à l'aube des récits sur le prophète, chantent et prient Les femmes de Médina K. avaient préparé pendant des jours les repas qu'elles apportèrent ensuite au village voisin. A l'occasion de cette fête, on abattit également une chèvre dans le village voisin. Le jour suivant, comme c'était l'habitude, on enleva les mauvaises herbes sur le champ du grand marabout. Malheureusement, je n'ai pas pu accepter l'invitation des villageois de les accompagner à la fête du Gamou (une partie de la population restait au village le matin de la fête), car je devais passer la soirée à Kaolack, un grand centre de la confrérie des Tidjanes. Cette ville est entre autre un nœud de communication important parce que la famille du grand marabout $\mathrm{El} \mathrm{Hadj} \mathrm{Abdoulaye} \mathrm{Niass} \mathrm{y} \mathrm{réside.} \mathrm{Son} \mathrm{successeur} \mathrm{El} \mathrm{Hadj} \mathrm{Ibrahima} \mathrm{Niass}$ joue un rôle clé dans les relations interafricaines et internationales, grâce à ses contacts multiples. Le soir du Gamou, la ville était pleine de croyants venus de l'extérieur. Durant toute la nuit, des hauts-parleurs éparpillés à travers toute la ville transmettaient des chants religieux soulignant ainsi l'ambiance solennelle. Avant et après l'évènement, la presse quotidienne sénégalaise (Le Soleil, Walfadjri... L'Aurore et Société) en a parlé en abondance.

En toutes circonstances, les villageois s'adressent, d'abord au marabout pour lui demander conseil. Moreau (1982: 244) décrit les différents rôles des marabouts de la manière suivante: "Progressivement, ces hommes sont devenus les concurrents des cadres de la société africaine; ensuite, ils ont eu tendance à prendre la place des guérisseurs, des devins, puis celle des sages et des responsables de l'initiation, enfin celle des chefs". Les responsables du groupement paysan, aussi, discutent de leurs projets avec l'Imam du village, ce qui veut dire qu'ils ont besoin de la légitimation par la religion.

Un mariage sans contrat? Organisations paysannes et l'Islam dans le processus de transformation au Sénégal

Les interpénétrations entre les responsables de l'organisation paysanne, les chefs traditionnels et les auto rites religieuses du village sont remarquables. Le président de l'organisation paysanne à Médina $\mathrm{G}$. est le petit-fils du fondateur du village. Après que les habitants du village eurent quitté l'ancienne implantation, le village fut abandonné 
pendant 55 ans. Maintenant donc, c'est O.N., le petit-fils du nouveau fondateur, qui préside actuellement le groupement paysan qui ,fonctionne depuis cinq ans sous forme de GIE. Le trésorier du groupement, qui est en même temps trésorier de l'association régionale ADAK (Association des Agriculteurs de la Région de Kaolack), appartient également à la famille N.. Dans le quartier du trésorier habite son frère qui, quant à lui, assure le fonctionnement de la deuxième école coranique. Un autre frère du trésorier, qui lui ressemble tellement qu'il pourrait être son frère jumeau, est l'Imam du village. Il habite dans la quartier voisin. Pendant mon séjour, l'Imam et le président du groupement rendaient régulièrement visite au président du groupement, non seulement pour prendre le thé, mais surtout pour établir de nouveaux projets. Ils ont ainsi p.ex. réfléchi sur un moyen de se procurer le plus vite possible de l'huile lorsque le moulin à mil dont le trésorier a la charge ne fonctionne plus. Après des pluies abondantes ne permettant pas le travail des champs, le trésorier de même que l'Imam s'arrêtaient souvent à la ferme du président, où j'habitais pendant mon séjour.

70 Médina G. est vraiment un cas typique pour analyser la liaison existant entre l'organisation paysanne et la confrérie. Dans les négociations officielles, les femmes ne jouent guère de rôle. Bien qu'il y ait aussi une présidente pour les femmes du groupement mixte, avec laquelle j'ai pu m'entretenir de ses tâches, aucun nom féminin ne figure dans les papiers officiels de l'immatriculation de l'organisation. Ce sont au contraire les hommes provenant ou ayant un lien de parenté avec ceux qui représentent les structures du pouvoir traditionnel (chef du village, Imam) qui se sont repartis les postes de responsabilité entre eux. Pour l'entente de Koungheul, Blundo (1994: pp. 107) relève pourquoi les femmes sont indispensables à la formation des groupes: "elles sont l'élément-clef de tout discours populiste qui se respecte (gouvernemental et non gouvernemental) et leur présence légitime donc l'existence de l'association. De plus, elles constituent une force de travail non négligeable...".

71 A cela s'ajoute le fait que les autres membres du groupement, eux aussi, cherchent régulièrement conseil auprès de l'Imam ou du marabout, afin d'avoir leurs assurances sur le plan religieux. A ce niveau, l'organisation paysanne a plutôt un rôle pratique, qui. tant qu'elle sert à améliorer la satisfaction des besoins fondamentaux, est considérée comme bon. La formation, cependant, n'est point considérée par toutes les autorités comme un besoin fondamental, de sorte que les opinions divergent à ce propos.

Quant à la règlementation des projets communs du village, on a pu observer une ébauche d'un procès de décalage. Alors qu'auparavant, un seul champ avait été cultivé pour la mosquée, et que son rapport financier servait pour les projets de la communauté villageoise (élevage de bœufs, construction d'un puits etc.), aujourd'hui on a ajouté un champ pour le groupement de paysans. Dans certains cas, ce dernier champ est plus grand, d'où également la récolte; ce qui constitue une bonne réserve pour le groupement villageois. La survie de la communauté villageoise pendant l'hivernage, avant la récolte donc, peut être assurée petit à petit de manière autonome. Parallèlement à ce déplacement de responsabilité, le champ pour la mosquée reste intact, continuant à symboliser la grande importance toujours accordée de la religion.

On ressent l'interpénétration de dogmes religieux avec la vie paysanne quotidienne lorsqu'on observe la conscience professionnelle. Aussi bien d'après le Coran que d'après les écrits de Cheikh Ahmadou Bamba et de Cheikh Tidjiane Sy, les croyants sont tenus à un travail incessant A côté de l'appel du fondateur des Mourides au travail continu, on souligne la mise en garde dans le Coran contre toute forme de cupidité (cf. Sourate 102, 
Le désir de s'enrichir). Cette éthique permet de concilier le travail sur le champ du marabout, avec celui sur le champ collectif. Les GIE orientés vers le profit ne vont pas dans un sens opposé à ces directives, car c'est la communauté qui bénéficie de ce profit. Comme ce profit est utilisé par les organisations paysannes pour de nouveaux investissements, on pourrait établir la thèse que les organisations paysannes profitent considérablement des motivations religieuses. Bayart (1993 : 303) se pose également la question de savoir, dans quelle mesure la religion peut aider les pays africains à entrer dans une nouvelle dynamique: "Au moment où l'Afrique noire s'enfonce dans une phase de régression économique et hésite entre la démocratie, la restauration autoritaire et la guerre, elle connaît une intense mobilisation religieuse dont la tonalité est moins explicitement politique que sociale et fidéiste".

74 En ce qui concerne l'interaction des paysans avec l'Etat, la question se pose, jusqu'à quel point les paysans ont la possibilité d'occuper l'espace libéré par l'Etat qui se retire. L'organisation paysanne s'est constituée dans les années 70 comme alternative aux coopératives, et cela en vue d'une autonomie, "mais elle n'allait pas dans le sens d'une démocratisation; la population n'obtint pas le pouvoir d'exploiter elle-même les coopératives" (Lachenmann 1991: 79) Déjà dans les années 60, à l'époque des coopératives, les systèmes traditionnels de pouvoir s'imposèrent : "Le cadre formel et la faiblesse de l'entreprise de l'administration sur le monde rural permettront aux marabouts d'investir, par le biais de leurs talibé, les institutions telles que les coopératives. Ils vont désormais, indirectement occuper les fonctions modernes" (Diop, Diouf 1992 : 76).

75 Néanmoins, la forte influence des confréries islamiques dans le secteur de l'arachide ne fonctionnait pas selon des principes de libre échange et menait donc à une stagnation. Selon Copans (1988: 98), la signification de la participation des marabouts à la production totale n'était jamais décisive: "Moyenne actuelle: 130000 tonnes pour 1884-1919, 407000 tonnes 1920-1939. Pour la période 1950-1969 cette moyenne s'élève à 656000 tonnes. Ce sont alors les beaux jours de l'exploitation coloniale et néo-coloniale... Pour les saisons 1952-53 et 1957-58, la part des mourides constitue exactement $25 \%$ de la production totale. Ce pourcentage est loin d'être insignifiant, mais il ne permet pas de conclure la domination de la confrérie mouride". Cependant, ce sont les Mourides qui ont permis la participation au marché mondial : "However, this dynamic has come to an end, integration within the world market is no longer of interest to the Senegalese state and national economy (exept for the problem of existing industrial transformation capacities), but peasants are - through their own efforts - unable to overcome what might be called the 'groundnut logic' on which their increasingly unstable existence is based" (Lachenmann 1994 :74).

76 Comme la dynamique de l'économie arachidière est désormais terminée, le rôle des Mourides se réduit, selon Copans (1988: 235), à un aspect immatériel : "Pour nous, la fonction déterminante des marabouts, qui apparaît de plus en plus comme telle, est d'ordre idéologique. Par ailleurs l'intervention grandissante de l'état dépossède directement ou indirectement, les marabouts de leurs prérogatives socio-politiques ou économiques". A cause de l'hégémonie économique, Croise O'Brien (1979: 226), lui aussi, décelait déjà à la fin des années 70 une nouvelle signification pour les Mourides en tant que premier syndicat autonome des paysans. Selon Croise O'Brien, les Mourides avaient une attitude intéressée, mais réelle pour la défense des droits des paysans. Entretemps, cela a changé de sorte que beaucoup de paysans se plaignent d'être 
négligés par les Mourides. Copans, quant à lui, écrit que le talibé ne peut compter que sur lui-même s'il veut améliorer sa situation (1988:235). Enfin Copans met en relief le mérite des Mourides concernant le maintien de l'unité nationale (1988: 257) "Aujourd'hui, la confrérie apparaît comme un médiateur nécessaire à cause de ses fonctions de contrôle et de mobilisation, ce qu'elle était jusqu'à présent, que comme l'un des seuls centres capables de produire une idéologie consubstantielle à l'ensemble des rapports sociaux sénégalais, c'est-à-dire une idéologie nationale". Vu le déclin de l'économie de l'arachide aujourd'hui, on peut se rendre compte que la constatation faite par Copans en 1979 - d'une perte de fonction - se vérifie certainement actuellement. On peut se demander tout de même si les Mourides ont effectivement réussi à développer une nouvelle idéologie ou si ce ne sont pas plutôt les organisations paysannes qui ont su créer un sentiment d'unité. D'après Diop et Diouf, les structures traditionnelles du pouvoir n'ont pas encore disparu, de sorte qu'ils parlent toujours de pression de la part de la clientèle politique rurale (1992: 86). De plus, à cause d'intérêts trop divergents, ils mettent en question le développement d'objectifs communs dans la politique agricole. (Voir les études empiriques à ce propos, comme la recherche de Lachenmann et al. 1990 et Lachenmann 1994).

Néanmoins, la possibilité existe que les efforts à la base soient reconnus au niveau gouvernemental, et qu'on en tienne compte aussi dans la politique agricole, dans celle du développement et dans la politique vis-à-vis des femmes. Jusqu'à présent, on peut constater que les groupes sociaux tels que les organisations paysannes s'inscrivent dans l'interaction entre l'Etat et les représentants religieux en formant un triangle dont la capacité à consolider ou à transformer les structures existantes reste à observer.

\section{BIBLIOGRAPHIE}

APROFES, s.d. : Association pour la promotion de la femme sénégalaise, Historique, But et objectifs, Partenaires à la base, Activités, Kaolack.

Bamba, Cheikh Ahmadou, s.d. : Mawaahibu Naafih, traduit par Papa K. Seck, Dakar, Lamp Fall.

Bayart, Jean-François (Ed.), 1993 : Religion et modernité politique en Afrique Noire. Dieu pour tous et chacun pour soi, Paris, Karthala.

Bello, Walden, Shea Cunningham, 1994 : "De l'ajustement structurel en ses implacables desseins", in : Le Monde Diplomatique (9), pp.8.

Blundo, Giorgio, 1991 : "La brique, la terre et le puits : administration locale, factionnalisme et auto-promotion au Sénégal", in : Kwan Kaï Hong (Ed.) : Jeux et enjeux de l'autopromotion. Vers d'autres formes de coopération au développement, Genf 1991, Cahiers de 1'I.U.E.D./Paris, Presses Universitaires de France, pp. 103-133.

Blundo, Giorgio, 1994 : "Le conflit dans l'Entente. Coopération et compétition dans les associations paysannes du bassin arachidier sénégalais", in : Jean-Pierre Jacob, Philippe Lavigne Delville, pp. 99-120. 
Blundo, Giorgio, 1995 : "Les courtiers du développement en milieu rural sénégalais", in : Cahiers d'Etudes Africaines, 137, XXXV-I, pp. 73-99.

Brandstetter, Anna Maria, Gerhard Grohs, Dieter Neubert (Ed.), 1994 : Afrika hilft sich selbst. Prozesse und Institutionen der Selbstorganisation, Münster, Lit- Verlag, Schriften der VAD, Band 13.

Bruchhaus, Eva-Maria, 1989 : "Réflexions des membres fondateurs d'une association paysanne au Sénégal par rapport à la crise de l'Économie monétaire internationale et les règles de l'islam", in : Développement et Coopération.

Cissokho, Mamadou, 1994 : "Die Foderation der Nicht-Regierungsorganisationen in Senegal (FONGS)", in : Anna Maria Brandstetter, Gerhard Grohs, Dieter Neubert (Ed.), pp. 42-51.

Copans, Jean, 1988 : Les marabouts de l'arachide. La confrérie mou ride et les paysans du Sénégal, Paris, L'Hannattan.

Coulon, Christian, Odile Reveyrand, 1990 : "L'Islam au féminin : Sokhna Magat Diop, Cheikh de la confrérie mouride (Sénégal)", Travaux et Documents 25, Bordeaux, Centre d'Étude d'Afrique Noire.

Coulon, Christian, 1983 : Les musulmans et le pouvoir en Afrique noire. Religion et contre-culture, Paris, Karthala.

Cruise O'Brien, Donal B., 1971 : The Mourides of Senegal. The Political and Economie Organization of an Islamic Brotherhood, Oxford, Clarendon Press.

Cruise O'Brien, Donal B., 1979 : "Ruling Class and Peasantry in Senegal ; 1906-1976 : The politics of a Monocrop Economy", in : R. Cruise O'Brien (Ed.), The political economy of underdevelopment. in Senegal, London, Sage.

Diop, Momar Coumba, Mamadou Diouf, 1992 : "L'administration sénégalaise, les confréries religieuses et les paysanneries", in : Afrique et Développement, vol. XVII (2).

Duruflé, Gilles, 1988 : L'ajustement structurel en Afrique (Sénégal, Côte d'Ivoire, Madagascar), Paris, Karthala.

Ebin, Victoria, 1993 : "Les commerçants mourides à Marseille et à New York. Regards sur les stratégies d'implantation", in : Emmanuel Grégoire et Pascal Labazée (Ed.) : Grands commerçants d'Afrique de l'Ouest. Logiques et pratiques d'un groupe d'hommes d'affaires contemporains, Paris, Karthala/Orstom.

Fall, Abdou Salam, 1994 : "Et si l'insertion urbaine passait par l'investissement dans des réseaux sociaux ? Réseaux formels et informels de solidarité et de dépendance dans les quartiers de Dakar", in : Jean-Pierre Jacob, Philippe Lavigne Delville (Ed.), pp. 292-303.

Fédération des Organisations non Gouvernementales du Sénégal. EO.N.G.S, s.d. : Action paysanne, Thiès.

Jacob, Jean-Pierre, Philippe Lavigne Delville (ss la dir. de), 1994 : Les associations paysannes en Afrique. Organisation et dynamiques, Paris, Karthala.

Lachenmann, Gudrun, 1991 : "Soziale Bewegungen als gesellschaftliche Kraft in dem Prozefl der Demokratisierung in Afrika? Bauemorganisationen im Senegal angesichts des Rückzugs des Staates und der Strukturanpassung", in : africa spectrum, 26. Jg., Nr. 1, pp. 73-97.

Lachenmann, Gudrun, 1992 : "Frauen als gesellschaftliche Kraft im sozialen Wandel in Afrika", in : Peripherie, 12. Jg., Nr. 47/48, pp. 74-93. 
Lachenmann, Gudrun, 1994 : "Civil Society and Social Movements in Africa", in : Jean-Pierre Jacob, Philippe Lavigne Delville, pp. 61-95.

Lachenmann, Gudrun, 1994 : "Einführung zur Lehrforschung. Transformationsprozesse in Westafrika (Senegal) und die Bedeutung der Bauembewegung", in : Gudrun Lachenmann et al. : Abschluflbericht Lehrforschung Westafrika, Universitiit Bielefeld, Forschungsschwerpunkt Entwicklungssoziologie, pp. 315-323.

Lachenmann, Gudrun, Matthias von Bechtolsheim, Sabine Becker, Türkan Karakurt, Jorg Meilicke, Claudia Pragua, 1990 : Organisations paysannes au Sénégal : comment renforcer les structures intermédiaires dans le processus de transformation socio-économique, Berlin, Institut allemand de développement

Loimaier, Roman, 1995 : "Sakularer Staat und Islam : Das Beispiel Senegal", in : Heidi Willer, Till Forster, Claudia Ortner-Buchberger (Ed.) : Macht der Identität - Identität der Macht. Prozesse und kultureller Wandel in Afrika, Münster, Lit.

Loimaier, Roman, 1994 : "Religios-okonomische Netzwerke in Senegal. Das Beispiel der muridischen Expansion in Dakar", in : Anna Maria Brandstetter, Gerhard Grohs, Dieter Neubert (Ed.), pp. 256-269.

Magassouba, Moriba, 1985 : L'Islam au Sénégal. Demain les mollahs ?, Paris, Karthala.

Marone, Ibrahima, 1970 : "Le tidjanisme au Sénégal", in : Bulletin de l'Institut Fondamental d'Afrique Noire, Série B, Sciences Humaines, (1), XXXII : 136-215.

Mbacké, Khadim, 1993 : Études islamiques 2. Islam et société, Dakar, IFAN.

Moreau, René Luc, 1982 : Africains Musulmans, Paris/Abidjan, Présence Africaine/Inades.

Olivier de Sardan, Jean-Pierre, Thomas Bierschenk, 1993 : "Les courtiers locaux du développement", in : Bulletin de l'APAD, N5, pp. 71-76.

République du Sénégal, Ministère de la femme, de l'enfant et de la famille, Comité national pour la préparation de Bejing 1995 (Ed.) : Rapport National sur les femmes. Lutte pour l'égalité, le développement et la paix, Dakar 1994.

République du Sénégal, Comité National de Concertation des Ruraux (C.N.C.R.) : Déclaration des Thiès, Thiès : 17.3.93.

Salzbrunn, Monika, 1994 : "Religion und Tradition' - Bauernorganisationen und islamische Bruderschaften im Transformationsprozefl im Senegal", in : Gudrun Lachenmann et al. : Abschlujlbericht Lehrforschung Westafrika, Universität Bielefeld, Forschungsschwerpunkt Entwicklungssoziologie, pp. 422-485.

Sy, Maïmouna, 1994 : Synthèse Atelier Paysan. Interview auprès des femmes, Kaolack, Deutsche Gesellschaft für Technische Zusammenarbeit, Projekt PAGERNA.

Villalon, Leonardo A., 1995 : Islamic Society and State Power in Senegal, Cambridge, Cambridge University Press.

Journaux :

Le Cafard libéré 1994 (338, 339, 341, 344, 345) ; Faits divers 1994 (10) ; Jeune Afrique 1994 (1753-1759) ; Promotion 1994 (226) ; Société ; le soleil 30.9.94 ; Sud quotidien 22.8.94, 30.9.94 ; le Témoin 1994 (214) ; La vache qui rit ? 1994 (148) ; Walfadjri... L'Aurore 3.7.94, 3.8.94, 8.8.94. 


\section{NOTES}

1.Pour l'étude intégrale, voir Salzbrunn, Monika, 1994 : Religion und "Tradition" Bauemorganisationen und islamische Bruderschaften im TransformationprozeB im Senegal, in : Gudnm Lachenmann et al. : AbschluBbericht Lehrforschung Westafrika, Universitat Bielefeld, Forschungsscbwerpunkt Entwicklungssoziologie. pp. 422-485.

2.Ebin 1993, in : Grégoire, Labazée : 101

3.Loimeier 1994, in : Brandstetter et al. (Ed.) : 256-269

4.Entretien du 15 août 1994 avec B. A. N., imam de Médina G.

5.Voir la bibliographie pour les journaux qui ont servi de source.

\section{AUTEUR}

MONIKA SALZBRUNN

Université de Bielefeld, Allemagne 